\title{
Comparação entre os métodos de Ziehl-Neelsen modificado e Acid-Fast-Trichrome para a pesquisa fecal de Cryptosporidium parvum e Isospora belli
}

\author{
Comparison between the modified Ziehl-Neelsen and Acid-Fast-Trichrome \\ methods for fecal screening of Cryptosporidium parvum and Isospora belli
}

\section{Carla R. Rigo' e Regina Maura B. Franco²}

\begin{abstract}
Resumo Devido a crescente importância dos coccídios intestinais (Cryptosporidium, Isospora e Cyclospora) como parasitos oportunistas, é fundamental para os laboratórios diferenciar morfologicamente estes protozoários; a técnica de Ziehl-Neelsen modificada (ZNm) é amplamente utilizada para este fim; recentemente, foi proposto um novo procedimento, a coloração combinada do ácido tricrômico (Acid-Fast-Trichrome - AFT). O objetivo do presente estudo foi comparar os processos AFT e ZNm para a detecção destes coccídios em amostras fecais de pacientes portadores do vírus VIH. Foram selecionados dois grupos de indivíduos, para inclusão no estudo, segundo a presença $(n=60)$ ou ausência de diarréia ( $n=60)$. As amostras de fezes foram coletadas em solução de formalina $10 \%$ e os esfregaços fecais preparados i) diretamente das fezes e ii) após concentração prévia a $500 \times$ xg (10 minutos), foram submetidos aos diferentes processos de coloração. Considerando-se a positividáde por técnica (AFT eZNM), verificou-se a superioridade do procedimento de $Z \mathrm{Nm}$ ( $n=19 ; 100 \%$ dos casos positivos) sobre o de AFT ( $n=8 ; 42,1 \%)$. Ambos possibilitaram a identificação dos 101 casos verdadeiramente negativos. Coccidiose intestinal foi mais frequente entre os pacientes que apresentaram diarréia $(26,6 \%)$ em comparação à positividade observada entre os indíviduos assintomáticos (5\%) sendo que C. cayetanensis não foi detectada em ambos os grupos. Foi de nosso interesse avaliar a aplicabilidade da técnica AFT para a coloracão deste protozoário. Devido à sensibilidade e especificidade obtida neste estudo (100\%), conclui-se que o método de ZNm continua sendo o mais indicado para o diagnóstico da criptosporidiose e isosporose, principalmente quando associado ao procedimento de centrífugo-concentração (500xg, 10 minutos). Embora a coloracão AFT tenha baixo custo, faz-se necessário o seu aperfeiçoamento pois este procedimento permite o diagnóstico simultâneo dos coccídios intestinais (C. parvum, I. belli e C. cayetanensis) e dos microsporídios.
\end{abstract}

Palavras-chaves: Coloração combinada do ácido tricrômico-AFT. Método de Ziehl-Neelsen modificado. Coccídios entéricos e VIH. Cryptosporidium. Isospora.

Abstract Due to the importance of Cryptosporidium parvum, Cyclospora cayetanensis and lsospora belli as opportunistic
parasites, it is essential for laboratories to morphologically differentiate these coccidia. Among the recommended
methods for diagnosis of C. parvum andl. belli is the Modified Ziehl-Neelsen stain (MZN) and recently a new technique,
the Acid-Fast-Trichrome (AFT) was proposed. The purpose of this study was to compare the AFT and MZN techniques
regarding the detection of C. parvum and I. belli oocysts in stool samples of HIV-positive patients. According to the
presence ( $n=60$ ) or absence of diarrhea ( $n=60$ ), two groups were selected for inclusion in this study. The stool samples
were collected in $10 \%$ formalin solution and the pellets, obtained without and after centrifugation-concentration (500xg;
10 min), were submitted to both stain techniques. Considering the positivity of the techniques (AFT and MZN), we
observed the superiority of MZN stain ( $n=19 ; 100 \%$ positive cases) over AFT (n=8; $42.1 \%$ ). The identification of the 101
truly-negative cases was possible with both techniques, but when the AFT stain was used, the oocysts showed a
variable inner stain degree, which resulted in false-negatives. Enteric coccidiosis was more frequent among the patients
who presented diarrhea (26.6\%) compared to asymptomatic individuals (5\%); C. cayetanensis was not detected in
these groups. We were interested in evaluating the employment of AFT for the Cyclospora oocyst stain. Based on the
sensitivity and specificity obtained in this study (100\%), we concluded that the MZN technique is still the most indicated
for routine use in clinical analysis laboratories, for the diagnosis of cryptosporidiosis and isosporosis, especially when
it was used together with the centrifugation procedure (500xg; 10 min). Our results showed that with some improvements,
AFT would be a simple and inexpensive technique appropriate for use in the diagnosis of intestinal coccidia (C. parvum,
C. cayetanensis and l. belli). It can also be extended to the detection of microsporidia. Key-words: Combined Acid-Fast-Trichrome Stain (AFT). Ziehl-Neelsen modified method. Enteric coccidia and HIV. Cryptosporidium. Isospora.

\footnotetext{
1. Bolsista de Iniciação Científica da Fundação de Amparo à Pesquisa do Estado de São Paulo (FAPESP). Processo n.‥ 98/12740-7. 2. Laboratório de Protozoologia do Departamento de Parasitologia do Instituto de Biologia da Universidade Estadual de Campinas, Campinas, SP. Endereço para correspondência: Dr ${ }^{\mathrm{a}}$. Regina Maura Bueno Franco. Dept ${ }^{\circ}$ de Parasitologia/IB/UNICAMP, Caixa Postal 6109, 13.083-970, Campinas, SP, Brasil. FAX: $55193289-3124$

e-mail: fllanos@uol.com.br

Recebido em 6/11/2000.
} 
Os indivíduos portadores do vírus VIH formam um segmento populacional altamente susceptível a desenvolver várias infeções oportunistas, cuja severidade é proporcional ao grau de imunossupressão ${ }^{312}$.

Como a diarréia aquosa é a principal manifestação clínica das infeções causadas pelos coccídios intestinais dos gêneros Cryptosporidium, Isospora e Cyclospora, o diagnóstico clínico não permite uma diferenciação específica do agente causal; a confirmação laboratorial constitui uma ferramenta imprescindível para estabelecer a diagnose diferencial e requer, no caso desses protozoários, o uso de técnicas específicas de concentração e coloração ${ }^{6}$.

Atualmente, os métodos de coloração ácidosresistentes são os mais recomendados para a demonstração dos oocistos de Cryptosporidium nas fezes ${ }^{4}$. Entretanto, Ignatius et $a^{\beta}$ descreveram um novo procedimento denominado Acid-Fast-Trichrome (AFT) que, segundo os autores, possibilita a detecção simultânea dos coccídios e microsporídios intestinais. Até o momento, a evidenciação desses parasitos em amostras fecais, só era possível com a utilização de colorações distintas, aplicáveis a cada agente parasitário ${ }^{5}$.

O interesse em aprimorar métodos de coloração é justificado tanto por sua fácil aplicação nos laboratórios de Análises Clínicas devido aos baixos custos do exame coproparasitológico como pelo fato de que o mesmo não é um procedimento invasivo, o que pode representar um impacto positivo na sobrevida dos hospedeiros imunocomprometidos, especialmente aqueles portadores da Síndrome de Imunodeficiência Adquirida.

No caso da coloração combinada do ácido tricrômico (AFT), ressalte-se que esse procedimento possibilita a evidenciação simultânea dos mais importantes protozoários emergentes (Cryptosporidium, Isospora e microsporídios), apenas utilizando microscopia óptica.

O presente estudo teve por objetivo tecer comparações entre as colorações Acid-Fast-Trichrome $(A F T)^{11}$ e de Ziehl-Neelsen modificada ${ }^{7}(\mathrm{ZNm})$, com o intuito de esclarecer vantagens inerentes a cada metodologia.

\section{MATERIAL E MÉTODOS}

Para a análise comparativa entre os diferentes procedimentos, foram empregadas amostras fecais de indivíduos portadores de infeção pelo VIH que: 1) relataram ocorrência de diarréia $(n=60)$ (definida como emissão de duas ou mais fezes líquidas em um período de 24 horas) e 2) não apresentaram diarréia ( $n=60)$.

Obtenção das amostras de fezes. As amostras fecais foram colhidas em solução de formalina $10 \%$ pelo setor de Parasitologia do Hospital de Clínicas e analisadas no laboratório de Protozoologia do Departamento de Parasitologia/IB/UNICAMP. Suspensão fecal contendo oocistos de Cyclospora cayetanensis foi cedida pelo Dr. Hércules de Moura (Center for Diseases Control, EUA).

Testes de concentração e coloração. Previamente, os espécimens fecais foram selecionados quanto à positividade para os coccídios intestinais (C. parvum, $C$. cayetanensis e I. belli) por meio dos métodos de Safranina Ácida ${ }^{15}$ e ZNm. Na seqüência, esfregaços preparados $i)$ diretamente das fezes preservadas em solução de formalina 10\% (1:3) e ii) após centrífugoconcentração a $500 \times \mathrm{xg}$ (10 minutos) com éter-PBS, foram submetidos aos diferentes procedimentos de coloração (AFT e ZNm) com o propósito de observar tanto a facilidade de evidenciação como a quantidade de oocistos encontrados. A leitura microscópica dos diversos preparados foi realizada sem que o examinador tivesse conhecimento do grupo ao qual cada indivíduo pertencia.

Posteriormente, foram efetuados testes com a finalidade de estabelecer o tempo ideal de coloração pelo método AFT ( $\left.\mathrm{t}_{1}=5 \mathrm{~min} ; \mathrm{t}_{2}=10 \mathrm{~min} ; \mathrm{t}_{3}=15 \mathrm{~min}\right)$.

Análise comparativa entre os processos de coloração. $\mathrm{Na}$ avaliação das lâminas, foram considerados os seguintes critérios: oocistos medindo em torno de 4-6um, formato esférico e presença de esporozoítos (=C. parvum); oocistos não esporulados, grandes, com 20-30 $\mu \mathrm{m}$ de tamanho (=I. belli); oocistos medindo em torno de $8-10 \mu \mathrm{m}$ e aspecto granuloso semelhante a mórula $(=C$. cayetanensis); contagem do número de oocistos em 50 campos microscópicos; intensidade da coloração dos oocistos; tonalidade de fundo da preparação; facilidade de visualização dos oocistos em aumento de 400x e dos esporozoítos; presença de oocistos que não sofreram impregnação adequada dos corantes. Estes fatores foram transformados em dados numéricos e, da pontuação final obtida, calculou-se a média, sendo este o valor usado para a comparação entre os procedimentos.

Análise estatística: os dados obtidos foram analisados utilizando-se o teste $t$ de Student para comparação de médias.

\section{RESULTADOS}

De um total de 120 amostras fecais incluídas no estudo, $19(15,8 \%)$ apresentaram positividade para os coccídios entéricos (C. parvum, l. belli) sendo que o procedimento de ZNm permitiu o encontro de oocistos em $100 \%$ dos casos $(n=19)$ e a coloração de AFT, em apenas $42,1 \%(n=8)$. As onze amostras consideradas como falso-negativas pelo método de AFT foram ZNm positivas. Ambos os procedimentos permitiram a identificação dos 101 casos negativos, ou seja, os verdadeiramente negativos (Tabela 1). A técnica de AFT apresentou sensibilidade de $42,1 \%$ e especificidade de 90,1\%; a de ZNm, alcançou índices de 100\%. Coccidiose intestinal foi mais freqüente entre os pacientes que apresentaram diarréia $(26,6 \%)$ em 
Tabela 1 - Comparacão entre os procedimentos de ZiehlNeelsen modificado (ZNm) e coloração combinada do ácido tricrômico (AFT) para detecção de Cryptosporidium parvum $e$ Isospora belli em amostras fecais.

\begin{tabular}{lccr}
\hline & \multicolumn{3}{c}{ ZNm } \\
\cline { 2 - 4 } AFT & positivo & negativo & total \\
\hline Positivo & 8 & 0 & 8 \\
Negativo & 11 & 101 & 112 \\
\hline Total & 19 & 101 & 120 \\
\hline
\end{tabular}

comparação à positividade observada entre os indivíduos assintomáticos $(5 \%)$ sendo esta diferença estatisticamente significativa $(t=3,376 ; p=0,001)$. Em ambos os grupos, não foi detectada C. cayetanensis (Tabela 2 ).

Quando considerada a quantidade de oocistos presentes nas preparações submetidas ao processo de centrífugo-concentraçăo, tais formas foram detectadas em maior número nos esfregaços submetidos à coloração de ZNm (Tabela 3) do que naqueles efetuados

Tabela 2 - Positividade para os coccídios intestinais em amostras fecais de pacientes portadores de VIH, apresentando ou não diarréia, atendidos pelo Hospital de Clínicas da UNICAMP.

\begin{tabular}{|c|c|c|c|c|}
\hline \multirow[b]{3}{*}{ Protozoário } & \multicolumn{4}{|c|}{ Pacientes portadores de VIH } \\
\hline & \multicolumn{2}{|c|}{ com diarréia } & \multicolumn{2}{|c|}{ sem diarréia } \\
\hline & № de positivos & $\%$ & № de positivos & $\%$ \\
\hline C. parvum & 11 & 18,3 & 1 & 1,6 \\
\hline I. belli & 4 & 6,6 & 1 & 1,6 \\
\hline C. cayetanensis & 0 & 0 & 0 & 0 \\
\hline Infecção concomitante & 1 & 1,6 & 1 & 1,6 \\
\hline Total & 16 & 26,6 & 3 & 5 \\
\hline
\end{tabular}

Tabela 3 - Número médio de oocistos (em 50 campos microscópicos) dos coccídios intestinais encontrados nos exames fecais efetuados por meio dos métodos de Ziehl-Neelsen modificado (ZNm) e coloração combinada do ácido tricrômico (AFT), sem e após concentração a 500xg (10 minutos).

\begin{tabular}{|c|c|c|c|c|c|c|c|c|}
\hline \multirow[b]{4}{*}{$\mathrm{AP}^{1}$} & & \multicolumn{6}{|c|}{ Técnica de Coloração } & \\
\hline & \multicolumn{4}{|c|}{$\mathrm{ZNm}$} & \multicolumn{4}{|c|}{ AFT } \\
\hline & \multicolumn{2}{|c|}{$\mathrm{C}^{*}$} & \multicolumn{2}{|c|}{$\mathrm{SC}^{\star *}$} & \multicolumn{2}{|c|}{$\mathrm{C}^{*}$} & \multicolumn{2}{|c|}{$S C^{\star \star}$} \\
\hline & média & $\mathrm{DP}^{2}$ & média & DP & média & DP & média & DP \\
\hline $1^{3}$ & 0,06 & 0,24 & 0 & 0 & 0 & 0 & 0 & 0 \\
\hline 2 & 0,62 & 0,57 & 0,24 & 0,43 & 0 & 0 & 0 & 0 \\
\hline 3 & 21,88 & 29,86 & 1,20 & 0,81 & 0 & 0 & 0,38 & 0,49 \\
\hline 4 & 8,76 & 6,83 & 1,54 & 0,79 & 5,04 & 6,63 & 0 & 0 \\
\hline 5 & 0,94 & 0,80 & 0 & 0 & 0,04 & 0,20 & 0 & 0 \\
\hline 6 & 3,80 & 5,69 & 1,42 & 2,19 & 0 & 0 & 0,04 & 1,14 \\
\hline 7 & 2,56 & 2,39 & 0,42 & 0,54 & 0 & 0 & 0 & 0 \\
\hline 8 & 0,68 & 0,71 & 0 & 0 & 0 & 0 & 0,18 & 0,52 \\
\hline 9 & 1,26 & 0,90 & 0,42 & 0,80 & 0 & 0 & 0 & 0 \\
\hline 10 & 0,02 & 0,14 & 0 & 0 & 0 & 0 & 0 & 0 \\
\hline 11 & 33,32 & 25,62 & 5,70 & 5,10 & 2,86 & 1,70 & 2,08 & 1,23 \\
\hline 12 & 1,24 & 0,43 & 0,34 & 0,51 & 0 & 0 & 0,38 & 0,48 \\
\hline 13 & 0,10 & 0,30 & 0 & 0 & 0 & 0 & 0 & 0 \\
\hline 14 & 0,10 & 0,30 & 0 & 0 & 0 & 0 & 0 & 0 \\
\hline $15^{4}$ & 0 & 0 & 0 & 0 & 0 & 0 & 0,04 & 0,20 \\
\hline 16 & 0,02 & 0,14 & 0 & 0 & 0 & 0 & 0 & 0 \\
\hline 17 & 0,10 & 0,30 & 0 & 0 & 0 & 0 & 0 & 0 \\
\hline 18 & 0,10 & 0,30 & 0 & 0 & 0 & 0 & 0 & () \\
\hline 19 & 0,20 & 0,40 & 0 & 0 & 0 & 0 & 0 & 0 \\
\hline 20 & 0,34 & 0,59 & 0,22 & 0,41 & 0,5 & 0,64 & 0,02 & 0,14 \\
\hline 21 & 0,10 & 0,30 & 0 & 0 & 0 & 0 & 0 & 0 \\
\hline
\end{tabular}

${ }^{1} \mathrm{AP}=$ amostra/paciente; ${ }^{2} \mathrm{DP}=$ desvio padrão; ${ }^{3}=$ amostras 1 a 14 : positividade para $C$. parvum;

${ }^{4}=$ amostras 15 a 21 : positividade para $I$. belli; ${ }^{*} \mathrm{C}=$ após concentração $(500 \times \mathrm{xg}, 10 \mathrm{~min})$; $\mathrm{SC}{ }^{\star *}=$ sem concentração préviamente à coloração. 
pela técnica AFT (ZNm: média final e desvio padrão após concentração: 18,6 $\pm 3,1 ;$ AFT: média final e desvio padrão após concentração: $10,1 \pm 2,3$ sendo as diferenças entre as médias estatisticamente significantes, com nível de significância de 5\%) e, quando analisados os esfregaços confeccionados sem enriquecimento prévio (Tabela 3 ), novamente o método de ZNm mostrou evidente superioridade (ZNm: média final e desvio padrão sem enriquecimento: $15,8 \pm 3,9$; AFT: média final e desvio padrão sem enriquecimento: $10,2 \pm 2,7$; sendo as diferenças entre as médias estatisticamente significantes; nível de significância de $5 \%$ ). Alterações no tempo de coloração (AFT), não acarretaram melhores resultados $\left(\mathrm{t}_{1}=5 \mathrm{~min}\right.$ - média final: 11,$3 ; t_{2}=10 \mathrm{~min}$ - média final: $17 ; t_{3}=15 \mathrm{~min}$ - média final: 17).

\section{DISCUSSÃO}

As prevalências observadas, neste estudo, para C. parvum (18,3\%) e I. belli $(6,6 \%)$, foram coerentes com a taxa anual de criptosporidiose (5 - 10\%) e isosporose $(15 \%)$ estimada para os indivíduos portadores de SIDA ${ }^{510}$ e que apresentam diarréia.

Neste estudo, verificou-se a superioridade da coloração de ZNm para o diagnóstico individual da criptosporidiose e isosporose (Tabela 1). Esta preparação propiciou uma melhor visualização dos oocistos que apresentavam diferentes tonalidades de róseo-avermelhado (Figura 1) e os esporozoítos eram facilmente observados na maioria dos oocistos presentes (Figuras 1 e 2). O fundo dos esfregaços apresentou tonalidade esverdeada ou azulada (dependendo da contra-coloração empregada), resultando num bom contraste. Também para I. belli, foi possível observar aspectos morfológicos (Figura 3).

Contrariamente, nas lâminas efetuadas pela técnica AFT, os oocistos apresentavam diferentes nuances de vermelho mas muitos não se impregnavam adequadamente com o corante (Figura 4), tornando difícil a evidenciação da morfologia interna para os protozoários estudados o que já havia sido mencionado por Ignatius et $a^{\beta}$. Outra desvantagem desta coloração é que ela oferece pouco contraste entre os oocistos e o fundo das preparações, predominando tons de cinza ou rosa claro dificultando a leitura dos espécimens, enquanto no caso do procedimento $\mathrm{ZNm}$, um técnico pode realizar a triagem do material utilizando menor aumento e facilmente identificar os oocistos róseos (Figura 5).

Neste estudo, foram empregadas amostras fecais contendo número variável de oocistos o que refletiu diferentes intensidades de infeção. Tal fato possibilitounos concluir que o procedimento ZNm é indicado para diagnóstico das infeções leves, quando o número de oocistos presentes nas fezes é baixo, como revelado pela contagem em 50 campos microscópicos (Tabela 3), devido à fácil visualização da morfologia interna.

O método de centrífugo-concentração em formalinaéter (500xg por 10 minutos) tem sido recomendado como o mais adequado para o diagnóstico das coccidioses entéricas quando associado às técnicas de coloração específicas $^{2}$. Alguns pesquisadores (comunicação pessoal) sugerem que não é necessário o emprego de métodos de concentração nos casos de infeção sintomática pois o simples exame das fezes diluídas (1:3) seria suficiente para um teste positivo. Entretanto, a técnica de concentração em formalina-éter utilizada nesta investigação acarretou um aumento da sensibilidade: em 12 casos (Tabela 3), só foi possível comprovar a presença de oocistos nas fezes após submetê-las ao procedimento prévio de concentração. Esses dados corroboram aqueles relatados por vários autores que só encontraram preparações ZNm positivas quando o grau de infecção era muito alto ${ }^{11}$. Além disto, seu uso é justificável pelo fato de que em um considerável número de pacientes imunodeficientes que apresentam diarréia crônica, C. parvum só pode ser detectado após o exame de múltiplas amostras ${ }^{1}$.

Resultados discrepantes entre as colorações (ZNm e AFT) em relação à detecção de $I$. belli podem ser explicados pela maior fragilidade dos oocistos à centrifugação; uma posterior avaliação dessas lâminas, revelou a presença de oocistos com morfologia alterada, dificultando sua evidenciação.

C. cayetanensis é um protozoário coccídio causador de diarréia crônica em pacientes imunocomprometidos; foi descrito como uma espécie nova a partir de $1993^{13}$. Seus oocistos $(8-10 \mu \mathrm{m})$ possuem morfologia e propriedades de coloração semelhantes aos de C. parvum (4-6um) o que justifica a atualização dos dados de prevalência de criptosporidiose.

O teste de autofluorescência tem sido recomendado para o diagnóstico de $C$. cayetanensis ${ }^{14}$ mas, esse critério pode não ser preciso pois relato recente ${ }^{14}$ enfatiza que os oocistos de $C$. parvum também podem ser autofluorescentes. Os testes laboratoriais referentes a aplicabilidade da coloração de AFT para evidenciação dos oocistos de Cyclospora permitiram concluir que o mesmo é adequado para a coloração das formas de resistência deste coccídio pois foram visíveis, nas lâminas, tanto as estruturas internas dos oocistos (que apresentaram tonalidade rosa, o que permite diferenciálos dos resíduos fecais) como a parede e os grânulos internos, embora a não impregnação do corante foi evientes em alguns oocistos (Figura 6).

Devido à sensibilidade e especificidade obtidas neste estudo (100\%), conclui-se i) que a técnica de ZNm continua sendo a mais indicada para uso rotineiro em laboratórios de análises clínicas, com relação ao diagnóstico da criptosporidiose e isosporose e ii) que a aplicação prévia de procedimento de centrífugoconcentração a $500 \times$ g (10 minutos) constitui um fator primordial para aumento da sensibilidade da coloração de Ziehl-Neelsen modificada. 


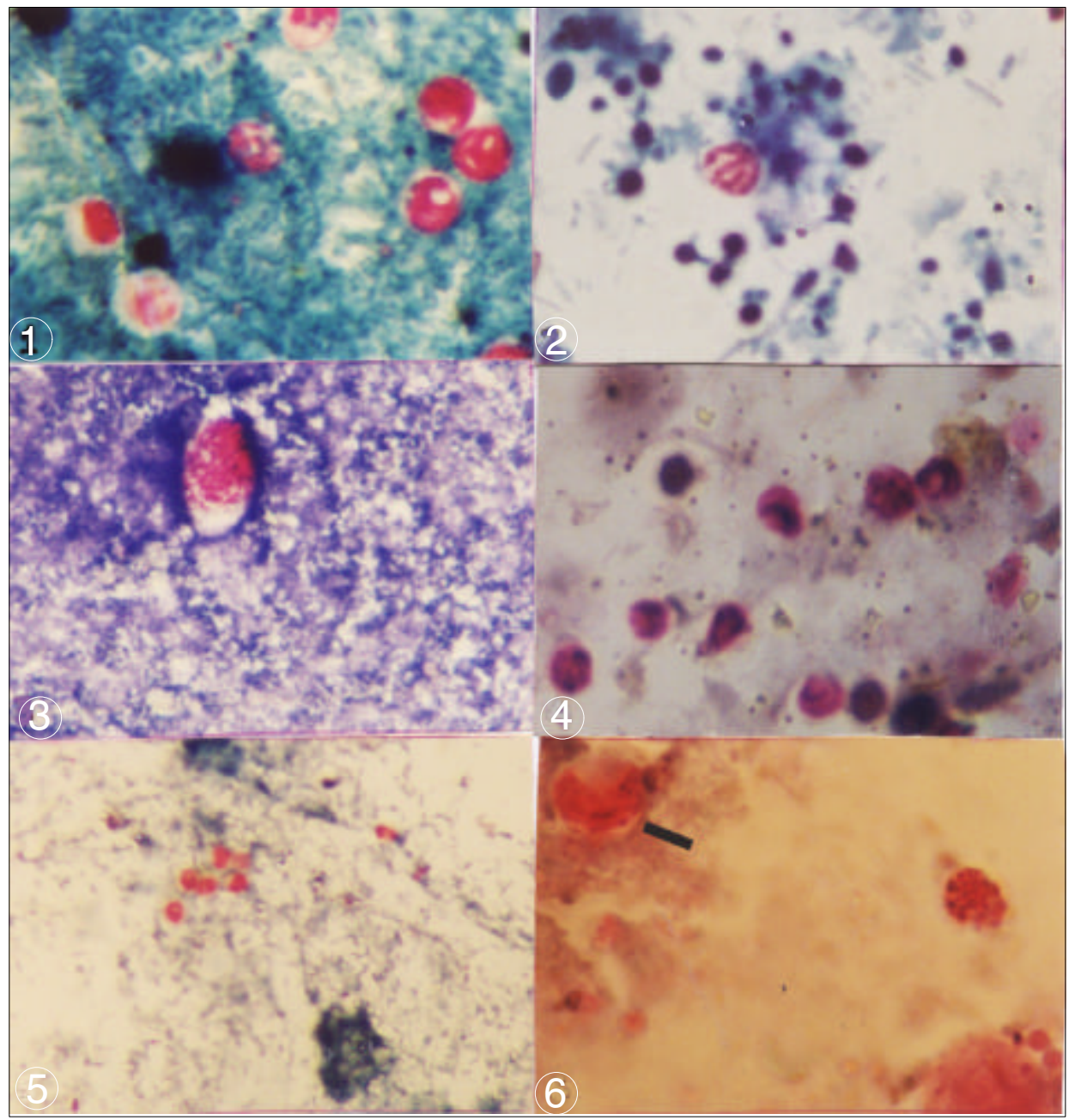

Figura 1 - Oocistos de Cryptosporidium parvum (coloração ZNm), aumento: 1000x. Figura 2 - Oocistos de Cryptosporidium parvum - 4 esporozoítos (coloracăo ZNM), aumento: 1000x. Figura 3 - Oocisto de Isospora belli (coloracão ZNm), aumento: 400x. Figura 4 -'Oocistos de Cryptosporidium parvum (coloracão AFT), aumento: 1000x. Figura 5 - Oocistos Cryptosporidium parvum (coloração ZNm), aumento: 400x. Figura 6 - Oocisto de Cyclospora cayetanensis (coloraçâo AFT), aumento: 1000x.

\section{AGRADECIMENTOS}

Agradecemos ao Dr. Hércules de Moura, Center for Diseases Control, por ter cedido os espécimens contendo Cyclospora cayetanensis, permitindo a realização dos testes de coloração e, aos biólogos Rubens Riscala Madi e Nilson Branco pelo auxílio na elaboração das figuras.

\section{REFERÊNCIAS BIBLIOGRÁFICAS}

1. Adal KA, Sterling CR, Guerrant RL. Cryptosporidium and Related Species. In: Blaser MJ, Smith PD, Ravdin, JI, Greenberg HB, Guerrant RL (eds) Infections of the Gastrointestinal Tract. Raven Press, New York, p. 1107-1128, 1995.

2. Clavel A, Arnal A, Sanchez E, Varea M, Quílez J, Ramírez I, Castilho, FJ. Comparison of 2 Centrifugation Procedures in the Formalin - Ethyl Acetate Stool Concentracion Technique for the Detection of Cryptosporidium oocysts. International Journal of Parasitology 26: 671-672,1996.

3. Curry A, Turner AJ, Lucas E. Opportunistic Protozoan Infections in Human Immunodeficiency Virus Disease: Review Highlighting
Diagnostic and Therapeutic Aspects. Journal of Clinical Pathology 44: 182-193, 1991.

4. De Carli GA. Parasitologia Clínica: Diagnóstico de Laboratório dos Coccídios e Microsporídios Intestinais. Cadernos Edipucrs; série Farmácia, Porto Alegre: ISBN 85-7430-102-107, 2000.

5. Goodgame RW. Understanding Intestinal Spore-Forming Protozoa: Cryptosporidia, Microsporidia, Isospora, and Cyclospora. Annals of Internal Medicine 124: 429-441, 1996.

6. Griffiths JK. Human Cryptosporidiosis: Epidemiology, Transmission, Clinical Disease, Treatment and Diagnosis. Advances in Parasitology 40: 38-73, 1998. 
7. Henriksen SA, Pohlenz JFL. Staining of Cryptosporidia by a Modified Ziehl-Neelsen Technique. Acta Veterinaria Scandinavia 22: $594-596,1981$.

8. Ignatius $R$, Eisenblatter $M$, Regnath $T$, Mansmann $U$, Futh $U$, Hahn H, Wagner J. Efficacy of Different Methods for Detection of Low Cryptosporidium parvum Oocysts Numbers or Antigen Concentrations in Stool Specimens. European Journal of Microbiology and Infectious Diseases 16: 732-736,1997.

9. Ignatius R, Lehmann M, Miksits K, Awand M, Engelmann E, Futh $\mathrm{U}$, Hahn H, Wagner J. A New Acid-Fast-Trichrome Stain for Simultaneous Detection of Cryptosporidium parvum and Microsporidial Species in Stool Specimens. Journal of Clinical Microbiology 35: 446-449, 1997.

10. Juranek DD. Cryptosporidiosis: Source of infection and Guidelines for Preventions. Clinical Infectious Diseases 21 (supl I): S57S61,1996

11. Kellogg JA, Elder CJ. Justification for Use of a Single Trichrome Stain as the Sole Means for Routine Detection of Intestinal
Parasites in Concentrated Stool Specimens. Journal of Clinical Microbiology 37: 835-837, 1999.

12. Smith PD. Intestinal Infectious in HIV-1 Disease. In: Blaser MJ, Smith PD, Ravdin JI, Greenberg HB, Guerrant RL (eds) Infections of the Gastrointestinal Tract. Raven Press, New York p. 483-493, 1995.

13. Soave R. Cyclospora: an Overview. Clinical Infectious Diseases 23: 429-437, 1996.

14. Varea M, Clavel A, Doiz O, Castilho FJ, Rubio MC, GómezLus R. Fuchsin fluorescence and autofluorescence in Cryptosporidium, Isospora and Cyclospora oocysts. International Journal of Parasitology 28: 1881-1883, 1998.

15. Visvesvara GS, Moura H, Kovacs-Nace E, Wallace S, Eberhard ML. Uniform Staining of Cyclospora Oocysts in Fecal Smears by a Modified Safranin Technique with Microwave Heating. Journal of Clinical Microbiology 35: 730733, 1997. 